\title{
The input work expression and the thermodynamics-based modelling framework for unsaturated expansive soils with double porosity
}

\author{
LI Jian $^{1}$, ZHAO ChengGang $^{1 *}$, CAI GuoQing ${ }^{1,2}$, ASREAZAD Saman $^{1}$, \\ XU Xi Frank ${ }^{1} \&$ HUANG QiDi ${ }^{1}$ \\ ${ }^{1}$ School of Civil Engineering, Beijing Jiaotong University, Beijing 100044, China; \\ ${ }^{2}$ ARC Centre of Encellence for Geotechnical Science and Engineering, The University of Newcastle, NSW 2308, Australia
}

Received November 23, 2012; accepted March 22, 2013

\begin{abstract}
In this paper, based on the porous media theory, the specific expressions of the total stress and the input work of unsaturated expansive soils with double porosity are proposed, and then the energy-conjugate variables are further derived. We make distinctions between the effects of capillary water and adsorbed water on the soil behaviour, as well as between the deformations of micro- and macrostructure. According to the derived input work expression and the work-energy-dissipation relations for an open multiphase thermodynamic system, we establish a thermodynamics-based modelling framework for unsaturated expansive soils with double porosity.
\end{abstract}

double porosity, unsaturated expansive soils, input work, energy-conjugate variables, constitutive model

Citation: $\quad$ Li J, Zhao C G, Cai G Q, et al. The input work expression and the thermodynamics-based modelling framework for unsaturated expansive soils with double porosity. Chin Sci Bull, 2013, 58: 3422-3429, doi: 10.1007/s11434-013-5828-9

Many geomaterials, such as fissured rocks [1], natural and compacted clays [2,3] and bentonite pellet mixtures [4], have a pore size distribution with at least two dominant values of porosity, or double porosity. The two scales of porosity correspond to the micropores (matrix pores, intraaggregates or intra-pellets) and macropores (fissures, interaggregate pore or inter-pellets). The research subject of this paper is unsaturated expansive soils which consist of two overlapping but distinct continua. The macrostructure refers to the arrangement of soil particle aggregates and the relatively large pores between them. The microstructure refers to clay particles and the micropores within them.

An important issue in the investigation of the behaviour of materials exhibiting two scales of porosity is the evolution of the internal structure and the proportional changes of micro- and macroporosity during the course of mechanical and wetting-drying stress paths. The compression tests $[5,6]$

*Corresponding author (email: cgzhao@bjtu.edu.cn) indicate that the macrostructural deformation is strongly affected by the mechanical loading of an aggregated soil while the corresponding change in the micropores is almost insignificant. The control-suction tests $[4,7]$ exhibit that the microstructure expands (or contracts) during wetting (or drying), and the microstructural volume change is reversible. However, the macrostructural deformation is always irreversible and is related to the initial compaction density, the variation range of suction, etc.

Based on the distinction between the deformations of micro- and macrostructure, Gens and Alonso [8] presented a framework for describing the behaviour of unsaturated expansive clays. Then Alonso et al. [9] further presented the stress-strain incremental relations (BExM). Mechanical coupling between both levels of structure was defined through two functions, one for wetting and the other for drying. Afterwards, a series of modifications and developments have been performed for the BExM [10-12].

Ziegler and Wehrli [13] presented an approach which is 
used to build constitutive models for general materials based on thermodynamic laws. This approach is further extended to building models of geomaterials $[14,15]$. Li $[16,17]$ deduced the expressions of input work and energy balance of unsaturated soils, and developed a thermodynamics-based modelling framework. Then the variation of gas pressure is further taken into account $[18,19]$.

In this paper, based on the porous media theory, the specific expressions of the total stress and the input work of unsaturated expansive soils with double porosity are proposed, and the energy-conjugate variables are further derived. According to the derived input work expression and the work-energy-dissipation relations for an open multiphase thermodynamic system, a thermodynamics-based modelling framework is also established.

\section{The characters of water in unsaturated expansive soils}

In the early $1950 \mathrm{~s}$, many researchers realized that an adequate understanding of the behaviour of unsaturated soils requires the proper consideration of matric suction. Actually matric suction (or potential) should be considered as the sum of two components, a capillary component and an adsorptive component [20,21]. The former one is related to air-water surface tension and negative water pressure, and is termed capillary suction. The capillary suction is always equal to $s_{\mathrm{c}}=p_{\mathrm{a}}-p_{\mathrm{w}}$, in which $p_{\mathrm{a}}$ and $p_{\mathrm{w}}$ are pore air pressure and pore water pressure, respectively. Capillary water which consists principally of bulk water arises from the surface tension mechanism. On the other hand, the latter one is related to the interaction of pore water and soil particle surfaces, i.e. physiochemical effect. Adsorbed water which is comprised of bound water arises from the physicochemical mechanism. Gens [22] figured out matric suction as a variable that expresses quantitatively the degree of attachment of the liquid to the solid phase. A large suction value refers to a very large potential of the water immediately adjacent to the solid surface, but it should not be viewed as pressures in the conventional bulk thermodynamic sense. However, the adsorptive component of matric suction and the physiochemical effect were always neglected in the earlier researches. Thus the validity of constitutive model of unsaturated soils based on capillary mechanism which is invalid at low water content, over the full range of degrees of saturation, is a question worth study.

The adsorptive component of the matric suction arises from two types of surface forces [20]. The first kind includes long-range electrostatic forces (e.g. diffuse double layer), short-range van der Waals and hydration forces. The second kind comprises of long-range forces due to the overlapping of two interfacial regions. Lu and Likos [23] indicated that the decrements in chemical potential due to surface force fields are much less at locations relatively far from particle surfaces than at locations near the surfaces. However, local thermodynamic equilibrium requires the chemical potential throughout the entire pore water phase to be the same. To satisfy this requirement, positive internal water pressure, which acts to increase the potential, builds up in the water films immediately adjacent to the soil particles.

As noted above, the capillary water and the adsorbed water have different properties. The effect of the latter on soil behaviour has to be considered when pore water is primarily retained on aggregate and platelet surfaces as water of hydration. The common stress variables, such as Bishop's stress, the double stress variables and the average soil skeleton stress, are only applicable to the situation that pore water is retained primarily as capillary mechanism. To take into consideration the adsorbed water effect, $\mathrm{Lu}$ and Likos [24] deduced an inter-particle stress $\sigma_{\mathrm{c}}$ which is expressed as

$$
\sigma_{\mathrm{c}}=\sigma-p_{\mathrm{a}}+\sigma_{\mathrm{pc}}+\sigma_{\text {cap }}+\left(p_{\mathrm{a}}-p_{\mathrm{w}}\right)\left[1-\left(A_{\mathrm{a}} / A\right)\right],
$$

where $\sigma$ is the total stress; $p_{\mathrm{a}}$ and $p_{\mathrm{w}}$ are pore air pressure and pore water pressure, respectively; $\sigma_{\mathrm{pc}}$ is the physicochemical interaction stress; $\sigma_{\text {cap }}$ is the capillary stress; $A$ is the projected area and $A_{\mathrm{a}}$ is the projected area where the air phase exists. The stress $\sigma_{\mathrm{c}}$ explicitly and effectively accounts for the effects of physicochemical stress and surface tension. However, it is inapplicable to the unsaturated porous continua with double porosity.

Borja and Koliji [25] used the first law of thermodynamics to derive an expression for an "effective", or constitutive, stress that is energy-conjugate to the rate of deformation of the solid matrix $\boldsymbol{d}$. The "effective" stress $\boldsymbol{\sigma}^{\prime}$ of unsaturated porous continua with double porosity has the form

$$
\sigma^{\prime \prime}=\sigma+B \bar{p} I,
$$

where $\sigma$ is the total Cauchy stress tensor; $B=1-\bar{K} / K_{s}$ is the Biot coefficient; $\bar{K}$ and $K_{\mathrm{s}}$ are the elastic bulk modulus of solid matrix and the intrinsic bulk modulus of solid phase, respectively; $\bar{p}=\psi^{\mathrm{m}} \bar{p}_{\mathrm{m}}+\psi^{\mathrm{M}} \bar{p}_{\mathrm{M}}$ is the mean pore fluid pressure for entire mixture; $\psi^{\alpha}(\alpha=\mathrm{m}, \mathrm{M})$ is the micro- or macropore fraction, and $\psi^{\mathrm{m}}+\psi^{\mathrm{M}}=1 ; \quad \bar{p}_{\alpha}=S^{\alpha \mathrm{w}} p_{\alpha \mathrm{w}}+\left(1-S^{\alpha \mathrm{w}}\right) p_{\alpha \mathrm{a}}$ is the mean micro- or macropore pressure; $S^{\alpha \mathrm{w}}$ is the microor macroporosity degree of saturation, and $S^{\alpha \mathrm{a}}=1-S^{\alpha \mathrm{w}} ; p_{\alpha \mathrm{w}}$ and $p_{\alpha \mathrm{a}}$ are pore water pressure and pore air pressure in micro- or macropores, respectively; $I$ is unit tensor of second-order. However, it is necessary to make distinctions between the deformations of the micro- and macrostructure, as well as the effects of the adsorbed water and the capillary water.

At the macroscopic level, the unsaturated expansive soils are composed of aggregates (ag), capillary water (c), gas (g) and adsorbed water (ad) phases [26]. The total surface of the aggregates is covered with the adsorbed water, with the 
capillary water concentrated at the corners, and the gas occupying the central portion of the pore. At the microscopic level, the aggregates are constituted of clay platelets and adsorbed water. Based on the porous media theory, the stress tensors of phases combine additively to form the total stress [25,27-29]

$$
\sigma_{\mathrm{ij}}=\sum_{\gamma=\mathrm{c}, \mathrm{g}}\left(n_{\mathrm{M}} S_{\gamma} u^{\gamma} \delta_{\mathrm{ij}}\right)+n_{\mathrm{M}} S_{\mathrm{ad}} p_{\mathrm{ij}}^{\mathrm{ad}}+\left(1-n_{\mathrm{M}}\right) s_{\mathrm{ij}},
$$

where $u^{\mathrm{c}}$ and $u^{\mathrm{g}}$ are the capillary water pressure and the pore air pressure, respectively; $p_{\mathrm{ij}}^{\text {ad }}$ is the adsorbed water stress, which is a second-order tensor because the adsorbed water can withstand a shear [20,30]; $s_{\mathrm{ij}}$ is the average stress in the aggregates; $n_{\mathrm{M}}$ is the macroporosity; $S_{\beta}(\beta=\mathrm{c}, \mathrm{g}, \mathrm{ad})$ is the degree of saturation of $\beta$ phase, and $\sum S_{\beta}=1$; and in the following, $\beta$ represents capillary water, adsorbed water and gas phases, and $\gamma$ represents capillary water and gas phases. The variables $S_{\mathrm{ad}}$ and $p_{\mathrm{ij}}^{\text {ad }}$ could not be explicitly determined. Nevertheless, by distinguishing the adsorbed water from the capillary water conceptually, a theoretical foundation is established for the following derivation.

\section{The expressions of the input work of unsatu- rated expansive soils with double porosity and the energy-conjugate variables}

Identifying the constitutive variables is the first step to deduce a constitutive model of unsaturated soils. The constitutive variables consist of stresses, strains, thermodynamics variables, etc. Based on the thermodynamics theory, the stress and strain variables can be chosen from the expression of input work of unsaturated soils [25,27,28].

At the macroscopic level, the unsaturated expansive soil is composed of aggregates, capillary water, gas and adsorbed water phases. Thus, the overall density $\rho$ is given by

$$
\rho=\sum_{\beta}\left(n_{\mathrm{M}} S_{\beta} \rho^{\beta}\right)+\left(1-n_{\mathrm{M}}\right) \rho^{\mathrm{ag}},
$$

where $\rho^{\mathrm{ag}}$ and $\rho^{\beta}$ are the densities of the aggregates and $\beta$ phase, respectively. The densities of adsorbed water and capillary water are approximately identical [30], thus we assume $\rho^{\mathrm{c}}=\rho^{\mathrm{ad}}=\rho^{\mathrm{w}}$.

The power input to a volume $V$ fixed in space, with a bounding area $A$, is the sum of the power input at the boundary and the power done by gravitational force:

$$
\begin{aligned}
& \int_{\mathrm{V}} W \mathrm{~d} V \\
& =-\int_{\mathrm{A}}\left[n_{\mathrm{M}} \sum_{\gamma}\left(S_{\gamma} u^{\gamma} f_{\mathrm{i}}^{\gamma} \delta_{\mathrm{ij}}\right)+n_{\mathrm{M}} S_{\mathrm{ad}} p_{\mathrm{ij}}^{\mathrm{ad}} f_{\mathrm{i}}^{\mathrm{ad}}+\left(1-n_{\mathrm{M}}\right) s_{\mathrm{ij}} v_{\mathrm{i}}\right] n_{\mathrm{j}} \mathrm{d} A \\
& \quad+\int_{\mathrm{V}}\left[n_{\mathrm{M}} \sum_{\beta}\left(S_{\beta} \rho^{\beta} f_{\mathrm{i}}^{\beta}\right)+\left(1-n_{\mathrm{M}}\right) \rho^{\mathrm{ag}} v_{\mathrm{i}}\right] g_{\mathrm{i}} \mathrm{d} V
\end{aligned}
$$

where the negative sign on the area integral arises from the compressive positive convention; $n_{\mathrm{i}}$ is the outward normal to the surface $A ; g_{\mathrm{i}}$ is the gravitational acceleration vector; $v_{\mathrm{i}}$ and $f_{\mathrm{i}}^{\beta}$ are the average velocities of the aggregates and $\beta$ phase, respectively; and the artificial seepage velocity of $\beta$ phase is defined by $w_{\mathrm{i}}^{\beta}=n_{\mathrm{M}} S_{\beta}\left(f_{\mathrm{i}}^{\beta}-v_{\mathrm{i}}\right)$. Substituting eqs.

(3) and (4) and the artificial seepage velocity of $\beta$ phase into eq. (5), and applying the divergence theorem of Gauss then leads to

$$
\begin{aligned}
\int_{\mathrm{V}} W \mathrm{~d} V= & \int_{\mathrm{V}}-\left[\sum_{\gamma}\left(u^{\gamma} w_{\mathrm{j}}^{\gamma}\right)+p_{\mathrm{ij}}^{\mathrm{ad}} w_{\mathrm{i}}^{\mathrm{ad}}+\sigma_{\mathrm{ij}} v_{\mathrm{i}}\right]_{, \mathrm{j}} \\
& +\left[\sum_{\beta}\left(\rho^{\beta} w_{\mathrm{i}}^{\beta}\right)+\rho v_{\mathrm{i}}\right] g_{\mathrm{i}} \mathrm{d} V,
\end{aligned}
$$

where a comma notation is used to indicate differentiation with respect to a spatial coordinate. Noting that the volume is arbitrary, it follows that the integrands on both sides of the equation are equal. Expanding the right-hand side then leads to

$$
W=-\sum_{\gamma}\left(u_{\mathrm{j}, \mathrm{j}}^{\prime \gamma} w_{\mathrm{j}}^{\gamma}+u^{\gamma} w_{\mathrm{j}, \mathrm{j}}^{\gamma}\right)-p_{\mathrm{ij}, \mathrm{j}}^{\mathrm{ad}} w_{\mathrm{i}}^{\mathrm{ad}}-p_{\mathrm{ij}}^{\mathrm{ad}} w_{\mathrm{i}, \mathrm{j}}^{\mathrm{ad}}-\sigma_{\mathrm{ij}} v_{\mathrm{i}, \mathrm{j}}
$$

where $u_{\mathrm{i}}^{\prime \gamma}$ and $p_{\mathrm{i}, \mathrm{j}, \mathrm{d}}^{\prime \text { ad }}$ are the excess pore pressure gradients in $\gamma$ phase and the adsorbed water, respectively. Here, it has been assumed that the total stress equilibrium is satisfied, i.e. $-\sigma_{\mathrm{ij}, \mathrm{j}}+\rho g_{\mathrm{i}}=0$.

The mass conservation equations of the aggregates, the capillary water, the adsorbed water and the gas phases can be expressed as

$$
\left\{\begin{array}{l}
\partial\left[\left(1-n_{\mathrm{M}}\right) \rho^{\mathrm{ag}}\right] / \partial t+\operatorname{div}\left[\left(1-n_{\mathrm{M}}\right) \rho^{\mathrm{ag}} v_{i}\right]=c^{\mathrm{ag}}, \\
\partial\left(n_{\mathrm{M}} S_{\mathrm{c}} \rho^{\mathrm{c}}\right) / \partial t+\operatorname{div}\left(n_{\mathrm{M}} S_{\mathrm{c}} \rho^{\mathrm{c}} f_{\mathrm{i}}^{\mathrm{c}}\right)=c^{\mathrm{c}}, \\
\partial\left(n_{\mathrm{M}} S_{\mathrm{ad}} \rho^{\mathrm{ad}}\right) / \partial t+\operatorname{div}\left(n_{\mathrm{M}} S_{\mathrm{ad}} \rho^{\mathrm{ad}} f_{\mathrm{i}}^{\mathrm{ad}}\right)=c^{\mathrm{ad}}, \\
\partial\left(n_{\mathrm{M}} S_{\mathrm{g}} \rho^{\mathrm{g}}\right) / \partial t+\operatorname{div}\left(n_{\mathrm{M}} S_{\mathrm{g}} \rho^{\mathrm{g}} f_{\mathrm{i}}^{\mathrm{g}}\right)=0,
\end{array}\right.
$$

where $c^{\mathrm{ag}}, c^{\mathrm{c}}$ and $c^{\mathrm{ad}}$ are the rates of mass exchange between the aggregates, the capillary water or the adsorbed water phases and other phases, respectively; and $c^{\text {ad }}$ is decomposed into $c^{\text {aa }}$ and $c^{\text {ac }}$, which are the rates of mass exchange between the adsorbed water phase and the aggregate phase or the capillary water phase, respectively. The equilibrium equations, $c^{\mathrm{ag}}+c^{\mathrm{aa}}=0$ and $c^{\mathrm{c}}+c^{\mathrm{ac}}=0$, are satisfied. Eq. (8) can be further expressed as

$$
\left\{\begin{array}{l}
v_{\mathrm{i}, \mathrm{i}}=\dot{n}_{\mathrm{M}} /\left(1-n_{\mathrm{M}}\right)-\dot{\rho}^{\mathrm{ag}} / \rho^{\mathrm{ag}}+c^{\mathrm{ag}} /\left[\left(1-n_{\mathrm{M}}\right) \rho^{\mathrm{ag}}\right], \\
f_{\mathrm{i}, \mathrm{i}}^{\mathrm{c}}=-\dot{n}_{\mathrm{M}} / n_{\mathrm{M}}-\dot{S}_{\mathrm{c}} / S_{\mathrm{c}}+c^{\mathrm{c}} /\left(n_{\mathrm{M}} S_{\mathrm{c}} \rho^{\mathrm{w}}\right), \\
f_{\mathrm{i}, \mathrm{i}}^{\mathrm{ad}}=-\dot{n}_{\mathrm{M}} / n_{\mathrm{M}}-\dot{S}_{\mathrm{ad}} / S_{\mathrm{ad}}+c^{\mathrm{ad}} /\left(n_{\mathrm{M}} S_{\mathrm{ad}} \rho^{\mathrm{w}}\right), \\
f_{\mathrm{i}, \mathrm{i}}^{\mathrm{g}}=-\dot{n}_{\mathrm{M}} / n_{\mathrm{M}}-\dot{S}_{\mathrm{g}} / S_{\mathrm{g}}-\dot{\rho}^{\mathrm{g}} / \rho^{\mathrm{g}} .
\end{array}\right.
$$

Here, it has been assumed that the spatial variations of 
$n_{\mathrm{M}}$ and $S_{\beta}$ are insignificant and the density of water is constant. Substituting the artificial velocities and noting that $v_{\mathrm{i}, \mathrm{i}}=-\dot{\varepsilon}_{\mathrm{ii}}$ leads to

$$
\left\{\begin{aligned}
w_{\mathrm{i}, \mathrm{i}}^{\mathrm{c}}= & S_{\mathrm{c}} \dot{\varepsilon}_{\mathrm{ii}}-n_{\mathrm{M}} \dot{S}_{\mathrm{c}}-S_{\mathrm{c}}\left(1-n_{\mathrm{M}}\right) \dot{\rho}^{\mathrm{ag}} / \rho^{\mathrm{ag}} \\
& +S_{\mathrm{c}} c^{\mathrm{ag}} / \rho^{\mathrm{ag}}+c^{\mathrm{c}} / \rho^{\mathrm{w}}, \\
w_{\mathrm{i}, \mathrm{i}}^{\mathrm{ad}}= & S_{\mathrm{ad}} \dot{\varepsilon}_{\mathrm{ii}}-n_{\mathrm{M}} \dot{S}_{\mathrm{ad}}-S_{\mathrm{ad}}\left(1-n_{\mathrm{M}}\right) \dot{\rho}^{\mathrm{ag}} / \rho^{\mathrm{ag}} \\
& +S_{\mathrm{ad}} c^{\mathrm{ag}} / \rho^{\mathrm{ag}}+c^{\mathrm{ad}} / \rho^{\mathrm{w}}, \\
w_{\mathrm{i}, \mathrm{i}}^{\mathrm{g}}= & S_{\mathrm{g}} \dot{\varepsilon}_{\mathrm{ii}}-n_{\mathrm{M}} \dot{S}_{\mathrm{g}}-S_{\mathrm{g}}\left(1-n_{\mathrm{M}}\right) \dot{\rho}^{\mathrm{ag}} / \rho^{\mathrm{ag}} \\
& +S_{\mathrm{g}} c^{\mathrm{ag}} / \rho^{\mathrm{ag}}-n_{\mathrm{M}} S_{\mathrm{g}} \dot{\rho}^{\mathrm{g}} / \rho^{\mathrm{g}} .
\end{aligned}\right.
$$

At the macroscopic level, the mass exchange between the aggregate phase and other phases leads to the density change of the aggregates which is given by

$$
\begin{aligned}
\frac{\partial \rho^{\mathrm{ag}}}{\partial t} & =\frac{1}{V^{\mathrm{ag}}} \frac{\partial m^{\mathrm{ag}}}{\partial t}-\frac{\rho^{\mathrm{ag}}}{V^{\mathrm{ag}}} \frac{\partial V^{\mathrm{ag}}}{\partial t}=\frac{V c^{\mathrm{ag}}}{V^{\mathrm{ag}}}+\rho^{\mathrm{ag}} \dot{\varepsilon}_{\mathrm{ii}}^{\mathrm{mm}} \\
& =\left(c^{\mathrm{ag}}+\rho^{\mathrm{ag}} \dot{\varepsilon}_{\mathrm{ii}}^{\mathrm{m}}\right) /\left(1-n_{\mathrm{M}}\right),
\end{aligned}
$$

where $\dot{\varepsilon}_{\mathrm{ii}}^{\mathrm{mm}}$ and $\dot{\varepsilon}_{\mathrm{ii}}^{\mathrm{m}}$ are the microstructural volumetric strain increment at the micro- and macroscopic level respectively, and the equilibrium equation, $\dot{\varepsilon}_{\mathrm{ii}}^{\mathrm{m}}=\left(1-n_{\mathrm{M}}\right) \dot{\varepsilon}_{\mathrm{ii}}^{\mathrm{mm}}$, is satisfied [4]; $m^{\text {ag }}$ and $V^{\text {ag }}$ are the quality and the volume of aggregates in the representative volume element, respectively. According to the method proposed by Alonso et al. [9], the total strain is the sum of the deformations of macro- and microstructure, i.e. $\varepsilon_{\mathrm{ij}}=\varepsilon_{\mathrm{ij}}^{\mathrm{M}}+\varepsilon_{\mathrm{kk}}^{\mathrm{m}} \delta_{i \mathrm{j}}$. Substituting the equilibrium equation, eqs. (10) and (11) to eq. (7), and noting that $-\sigma_{\mathrm{ij}} v_{\mathrm{i}, \mathrm{j}}=\sigma_{\mathrm{ij}} \dot{\varepsilon}_{\mathrm{ij}}$, it yields the work input to unsaturated expansive soils with double porosity as

$$
\begin{aligned}
W= & -u_{\mathrm{j}}^{\prime \mathrm{c}} w_{\mathrm{j}}^{\mathrm{c}}-u_{\mathrm{j} j}^{\mathrm{g}} w_{\mathrm{j}}^{\mathrm{g}}-p_{\mathrm{ij}, \mathrm{j}}^{\mathrm{ad}} w_{\mathrm{i}}^{\mathrm{ad}} \\
& +\left(\sigma_{\mathrm{ij}}-S_{\mathrm{g}} u^{\mathrm{g}} \delta_{\mathrm{ij}}-S_{\mathrm{c}} u^{\mathrm{c}} \delta_{\mathrm{ij}}-S_{\mathrm{ad}} p_{\mathrm{ij}}^{\mathrm{ad}}\right) \dot{\varepsilon}_{\mathrm{ij}}^{\mathrm{M}}-n_{\mathrm{M}}\left(u^{\mathrm{g}}-u^{\mathrm{c}}\right) \dot{S}_{\mathrm{c}} \\
& -n_{\mathrm{M}}\left(u^{\mathrm{g}}-p_{\mathrm{ij}}^{\mathrm{ad}} \delta_{\mathrm{ij}}\right) \dot{S}_{\mathrm{ad}}-\left(u^{\mathrm{c}}-p_{\mathrm{ij}}^{\mathrm{ad}} \delta_{\mathrm{ij}}\right) c^{\mathrm{c}} / \rho^{\mathrm{w}} \\
& -p_{\mathrm{ij}}^{\mathrm{ad}} \delta_{\mathrm{ij}} c^{\mathrm{aa}} / \rho^{\mathrm{w}}+\sigma_{\mathrm{ij}} \delta_{\mathrm{ij}} \dot{\varepsilon}_{\mathrm{kk}}^{\mathrm{m}}+u^{\mathrm{g}} n_{\mathrm{M}} S_{\mathrm{g}} \dot{\rho}^{\mathrm{g}} / \rho^{g} .
\end{aligned}
$$

The first three terms on the left side of the equation is the work caused by the seepage of the pore fluids relative to the aggregates; the fourth term is the work caused by the deformation of the macrostructure; the fifth and sixth terms are the work caused by the volume change of the capillary water and the adsorbed water, respectively; the seventh term is the work caused by the mass exchange between the capillary water phase and the adsorbed water phase; the eighth term is the work caused by the mass exchange between the adsorbed water phase and the aggregate phase; the ninth term is the work caused by the deformation of the microstructure; the tenth is the work caused by the compression of the gas phase. Houlsby [27], Zhao et al. [28] and Borja and Koliji [25] have developed the expression of the work input of unsaturated soils and unsaturated porous continua with double porosity, respectively, which are different from eq. (12) . The interpretations of the key terms in eq. (12) are as follows.

Firstly, the forth term in eq. (12) can be further expressed as

$$
\left[\left(\sigma_{\mathrm{ij}}-u^{g} \delta_{\mathrm{ij}}\right)+S_{\mathrm{M}} s_{\mathrm{c}} \delta_{\mathrm{ij}}-S_{\mathrm{ad}}\left(p_{\mathrm{ij}}^{\mathrm{ad}}-u^{\mathrm{c}} \delta_{\mathrm{ij}}\right)\right] \dot{\varepsilon}_{\mathrm{ij}}^{\mathrm{M}}=\hat{\sigma}_{\mathrm{ij}} \dot{\varepsilon}_{\mathrm{ij}}^{\mathrm{M}},
$$

where $\hat{\sigma}_{\mathrm{ij}}=\left(\sigma_{\mathrm{ij}}-u^{\mathrm{g}} \delta_{\mathrm{ij}}\right)+S_{\mathrm{M}} s_{\mathrm{c}} \delta_{\mathrm{ij}}-S_{\mathrm{ad}}\left(p_{\mathrm{ij}}^{\mathrm{ad}}-u^{\mathrm{c}} \delta_{\mathrm{ij}}\right)$ is the stress variable which is work conjugate to the macroscopic strain; $s_{\mathrm{c}}=u^{\mathrm{g}}-u^{\mathrm{c}}$ is the capillary suction; $S_{\mathrm{M}}=S_{\mathrm{c}}+S_{\mathrm{ad}}$ is the macroporosity degree of saturation. The stress $\hat{\sigma}_{\mathrm{ij}}$ is termed inter-aggregate stress which explicitly and effectively accounts for the inter-aggregate stress due to physicochemical mechanism. The expression of $\hat{\sigma}_{\mathrm{ij}}$ can be simplified according to the water content. At high water content levels, pore water is retained primarily as capillary menisci located between aggregates. Thus, the amount of adsorbed water and the physiochemical effect can be ignored. At low water content levels, pore water is primarily retained on aggregate and platelet surfaces as water of hydration. Thus the effect of the effect of the physiochemical effect is significant.

Secondly, the terms from the fifth to the seventh are the work caused by the structure change of the pore fluids. They can be further expressed by

$$
-\tilde{S}_{\mathrm{c}} \dot{S}_{\mathrm{M}}-\left(u^{\mathrm{c}}-p_{\mathrm{ij}}^{\mathrm{ad}} \delta_{\mathrm{ij}}\right)\left(n_{\mathrm{M}} \dot{S}_{\mathrm{ad}}-c^{\mathrm{ac}} / \rho^{\mathrm{w}}\right)
$$

where $\tilde{s}_{\mathrm{c}}=n_{\mathrm{M}}\left(u^{\mathrm{g}}-u^{\mathrm{c}}\right)$ is the modify capillary suction. The first term in eq. (14) demonstrates that the strain-like quantity, work conjugate to $\tilde{s}_{\mathrm{c}}$, is the increment of macroporosity degree of saturation $\dot{S}_{\mathrm{M}}$. The second term in eq. (14) indicates that the quantity, work conjugate to the volume change of the adsorbed water, is the stress variable $u^{\mathrm{c}}-p_{\mathrm{ij}}^{\mathrm{ad}} \delta_{\mathrm{ij}}$. Similarly, Derjaguin et al. [20] defined the disjoining pressure as the difference between a normal component of adsorbed water pressure and bulk liquid phase pressure. However, the adsorbed water and the bulk liquid phase have the same chemical potential.

Thirdly, the eighth and ninth terms are the work caused by the deformation of the microstructure. At the macroscopic level, the mass exchange between the aggregate phase and the adsorbed water phase leads to the microstructural volume change. Thus the micro stain can be expressed as

$$
\dot{\varepsilon}_{\mathrm{kk}}^{\mathrm{m}}=-\dot{V}^{\mathrm{ag}} / V=-\left(c^{\mathrm{aa}} / \rho^{\mathrm{w}}\right) V / V=c^{\mathrm{aa}} / \rho^{\mathrm{w}} .
$$

Substituting eq. (15) into the eighth and ninth terms in eq. (12) yields

$$
\left(\sigma_{\mathrm{ij}}-p_{\mathrm{ij}}^{\mathrm{ad}}\right) \delta_{\mathrm{ij}} \dot{\varepsilon}_{\mathrm{kk}}^{\mathrm{m}}=\hat{\sigma}_{\mathrm{ii}} \dot{\varepsilon}_{\mathrm{kk}}^{\mathrm{m}},
$$


where $\hat{\sigma}_{\mathrm{ii}}=\left(\sigma_{\mathrm{ij}}-p_{\mathrm{ij}}^{\mathrm{ad}}\right) \delta_{\mathrm{ij}}$ is the stress variable that is work conjugate to the microstructural volume change.

\section{The thermodynamics-based modelling frame- work of unsaturated expansive soils with double porosity}

Based on the derived input work equation and the workenergy-dissipation relations for an open multiphase thermodynamic system, we can develop a thermodynamicsbased modelling framework for unsaturated expansive soils with double porosity. To simplify eq. (12), we make the main assumptions as follows:

(i) the work caused by the seepage of the pore fluids relative to the aggregates is ignored [16], so the first three terms of eq. (12) are zero;

(ii) the work caused by the volume change of the adsorbed water is ignored, so the second term of eq. (14) is zero.

Based on these assumptions, eq. (12) can be expressed as

$$
W=\hat{\sigma}: \dot{\boldsymbol{\varepsilon}}^{\mathrm{M}}-\tilde{s}_{\mathrm{c}} \dot{S}_{\mathrm{M}}+u^{\mathrm{g}} n_{\mathrm{M}} S_{\mathrm{g}}\left(\dot{\rho}^{\mathrm{g}} / \rho^{\mathrm{g}}\right)+\hat{\sigma} \dot{\varepsilon}^{\mathrm{m}} .
$$

The Clausius-Duhem inequality yields the local dissipation function $D$

$$
D=W-\mathrm{d} \psi \geqslant 0,
$$

where $\psi$ is the Helmholtz function. According to the method suggested by Rice [32], the local dissipation function can be expressed as the product of a set of imposed thermodynamic forces $\zeta_{\mathrm{i}}$ and a set of increments of internal variables $\delta \xi_{\mathrm{i}}$ which are the dual of the former, i.e. $D=\zeta_{\mathrm{i}} \delta \xi_{\mathrm{i}}$. Substituting eq. (17) into eq. (18) yields the increment of the Helmholtz function

$$
\mathrm{d} \psi=\hat{\sigma}: \dot{\boldsymbol{\varepsilon}}^{\mathrm{M}}-\tilde{s}_{\mathrm{c}} \dot{S}_{\mathrm{M}}+u^{\mathrm{g}} n_{\mathrm{M}} S_{\mathrm{g}}\left(\dot{\rho}^{\mathrm{g}} / \rho^{\mathrm{g}}\right)+\hat{\sigma} \dot{\varepsilon}^{\mathrm{m}}-\zeta_{\mathrm{i}} \delta \xi_{\mathrm{i}} .
$$

The dissipation in a soil is attributable to its internal structural rearrangements. The pattern of internal rearrangements in an unsaturated soil can be denoted symbolically as [32]

$$
H=H\left(H_{\mathrm{M}}, H_{\mathrm{f}}\right),
$$

which states that the internal structural rearrangements in unsaturated soil can be decomposed into two parts, i.e. $H_{\mathrm{M}}$ and $H_{\mathrm{f}}$. The former denotes the pattern of the macrostructural rearrangements, and the latter denotes that in connection with the dissipation in the pore fluids. In this paper, we attempt to take into consideration the interactions between water rentenlion behaviour and mechanical behaviour [16,18], as well as the microstructural volume change and the deformation of macrostructure. Alonso et al. [9] demonstrated the micro-macrostructural interaction mechanisms as follow: the micro- and macrostructure expand during wetting at low confining stress, however the macropores are invaded by the aggregates at high confining stress; in contrast, the mi- cro- and macrostructure contract during drying at loose state, however the macropores develop as a result of drying at dense state. Thus, $H_{\mathrm{M}}$ and $H_{\mathrm{f}}$ can be further expressed symbolically as

$$
\left\{\begin{array}{l}
H_{\mathrm{M}}=H_{\mathrm{M}}\left(H_{\mathrm{M}}^{\prime}, H_{\mathrm{M}}^{\prime \prime}, H_{\mathrm{M}}^{\prime \prime \prime}\right), \\
H_{\mathrm{f}}=H_{\mathrm{f}}\left(H_{\mathrm{f}}^{\prime}, H_{\mathrm{f}}^{\prime \prime}\right),
\end{array}\right.
$$

where $H_{\mathrm{M}}^{\prime}, H_{\mathrm{M}}^{\prime \prime}$ and $H_{\mathrm{M}}^{\prime \prime \prime}$ denote the macrostructural rearrangement due to the change of the inter-aggregate stress, the plastic increment of macroporosity degree of saturation and the microstructural deformation, respectively; $H_{\mathrm{f}}^{\prime}$ and $H_{\mathrm{f}}^{\prime \prime}$ denote the dissipation in the pore fluids due to the capillary suction and the macrostructural plastic strain. They can be expressed, again symbolically, by

$$
\left\{\begin{array}{l}
\delta H_{\mathrm{M}}^{\prime} \propto\left\langle L_{\mathrm{M}}\right\rangle \\
\delta H_{\mathrm{M}}^{\prime \prime} \propto \delta S_{\mathrm{M}}^{\mathrm{p}} \\
\delta H_{\mathrm{M}}^{\prime \prime \prime} \propto \mathrm{d} \varepsilon^{\mathrm{m}}
\end{array}, \quad\left\{\begin{array}{l}
\delta H_{\mathrm{f}}^{\prime} \propto\left\langle L_{\mathrm{f}}\right\rangle \\
\delta H_{\mathrm{f}}^{\prime \prime} \propto \delta \varepsilon^{\mathrm{Mp}},
\end{array}\right.\right.
$$

where $L_{\alpha}$ is known as the loading index for the soil skeleton or the pore fluids; $\langle\cdot\rangle$ is the McCauley brackets such that the $\left\langle L_{\alpha}\right\rangle$ is a non-negative factor; $\delta \varepsilon^{\mathrm{Mp}}$ is the macrostructural plastic strain increment; $\delta S_{\mathrm{M}}^{\mathrm{p}}$ is the plastic increment of macroporosity degree of saturation.

When the interphase exchanges in entropy are negligible, the dissipation in an unsaturated soil can be additively decomposed as

$$
\zeta_{\mathrm{i}} \delta \xi_{\mathrm{i}}=\zeta_{\mathrm{i}}^{\mathrm{M}} \delta \xi_{\mathrm{i}}^{\mathrm{M}}+\zeta_{\mathrm{i}}^{\mathrm{f}} \delta \xi_{\mathrm{i}}^{\mathrm{f}}
$$

Because the change of the internal structure is fully described by $\delta \xi_{i}^{\alpha}$, the symbolic expressions are given by

$$
\left\{\begin{array}{l}
\delta \xi_{\mathrm{i}}^{\mathrm{M}} \propto \delta H_{\mathrm{M}}^{\prime}, \\
\delta \xi_{\mathrm{i}}^{\mathrm{f}} \propto \delta H_{\mathrm{f}}^{\prime} .
\end{array}\right.
$$

When the interphase exchanges in internal energy and entropy are negligible, the change in free energy in an unsaturated soil can be additively decomposed as

$$
\mathrm{d} \psi=\mathrm{d} \psi_{\mathrm{M}}+\mathrm{d} \psi_{\mathrm{f}}+\mathrm{d} \psi_{\mathrm{g}}+\mathrm{d} \psi_{\mathrm{m}},
$$

where

$$
\left\{\begin{array}{l}
\mathrm{d} \psi_{\mathrm{M}}\left(\varepsilon^{\mathrm{M}}, H_{\mathrm{M}}\right)=\hat{\sigma}: \mathrm{d} \varepsilon^{\mathrm{M}}-\zeta_{\mathrm{i}}^{\mathrm{M}} \delta \xi_{\mathrm{i}}^{\mathrm{M}}, \\
\mathrm{d} \psi_{\mathrm{f}}\left(S_{\mathrm{M}}, H_{\mathrm{f}}\right)=-\tilde{s}_{\mathrm{c}} \mathrm{d} S_{\mathrm{M}}-\zeta_{\mathrm{i}}^{\mathrm{f}} \delta \xi_{\mathrm{i}}^{\mathrm{f}}, \\
\mathrm{d} \psi_{\mathrm{g}}\left(\rho_{\mathrm{g}}\right)=n_{\mathrm{M}}\left(1-S_{\mathrm{M}}\right) p_{\mathrm{g}} \mathrm{d}\left(\ln \rho_{\mathrm{g}}\right), \\
\mathrm{d} \psi_{\mathrm{m}}\left(\varepsilon^{\mathrm{m}}\right)=\hat{\sigma} \mathrm{d} \varepsilon^{\mathrm{m}} .
\end{array}\right.
$$

Note that $\mathrm{d} \psi_{\mathrm{g}}$ and $\mathrm{d} \psi_{\mathrm{m}}$ do not include dissipation terms because any changes in air density and microstructural volume are reversible. For convenience the Gibbs functions are further defined by the following Legendre transforms of $\psi_{\mathrm{M}}, \psi_{\mathrm{f}}$ and $\psi_{\mathrm{m}}$ : 


$$
\left\{\begin{array}{l}
\phi_{\mathrm{M}}\left(\hat{\boldsymbol{\sigma}}, H_{\mathrm{M}}\right)=\hat{\boldsymbol{\sigma}}: \boldsymbol{\varepsilon}^{\mathrm{M}}-\psi_{\mathrm{M}}, \\
\phi_{\mathrm{f}}\left(\tilde{s}_{\mathrm{c}}, H_{\mathrm{f}}\right)=-\tilde{s}_{\mathrm{c}} S_{\mathrm{M}}-\psi_{\mathrm{f}}, \\
\phi_{\mathrm{m}}(\hat{\sigma})=\hat{\sigma} \varepsilon^{\mathrm{m}}-\psi_{\mathrm{m}},
\end{array}\right.
$$

with their increments given by

$$
\left\{\begin{aligned}
\mathrm{d} \phi_{\mathrm{M}}\left(\hat{\boldsymbol{\sigma}}, H_{\mathrm{M}}\right) & =\boldsymbol{\varepsilon}^{\mathrm{M}}: \mathrm{d} \hat{\boldsymbol{\sigma}}+\zeta_{\mathrm{i}}^{\mathrm{M}} \delta \xi_{\mathrm{i}}^{\mathrm{M}} \\
& =\left(\partial \phi_{\mathrm{M}} / \partial \hat{\boldsymbol{\sigma}}\right): \mathrm{d} \hat{\boldsymbol{\sigma}}+\zeta_{\mathrm{i}}^{\mathrm{M}}\left(\hat{\boldsymbol{\sigma}}, H_{\mathrm{M}}\right) \delta \xi_{\mathrm{i}}^{\mathrm{M}}, \\
\mathrm{d} \phi_{\mathrm{f}}\left(\tilde{s}_{\mathrm{c}}, H_{\mathrm{f}}\right) & =-S_{\mathrm{M}} \mathrm{d} \tilde{s}_{\mathrm{c}}+\zeta_{\mathrm{i}}^{\mathrm{f}} \delta \xi_{\mathrm{i}}^{\mathrm{f}} \\
& =\left(\partial \phi_{\mathrm{f}} / \partial \tilde{s}_{\mathrm{c}}\right) \mathrm{d} \tilde{s}_{\mathrm{c}}+\zeta_{\mathrm{i}}^{\mathrm{f}}\left(\tilde{s}_{\mathrm{c}}, H_{\mathrm{f}}\right) \delta \xi_{\mathrm{i}}^{\mathrm{f}}, \\
\mathrm{d} \phi_{\mathrm{m}}(\hat{\sigma}) & =\varepsilon^{\mathrm{m}} \mathrm{d} \hat{\sigma} .
\end{aligned}\right.
$$

Eq. (28) indicates that the general strains are given by

$$
\left\{\begin{array}{l}
\boldsymbol{\varepsilon}^{\mathrm{M}}=\partial \phi_{\mathrm{M}}\left(\hat{\boldsymbol{\sigma}}, H_{\mathrm{M}}\right) / \partial \hat{\boldsymbol{\sigma}} \\
-S_{\mathrm{M}}=\partial \phi_{\mathrm{f}}\left(\tilde{s}_{\mathrm{c}}, H_{\mathrm{f}}\right) / \partial \tilde{s}_{\mathrm{c}} \\
\varepsilon^{\mathrm{m}}=\partial \phi_{\mathrm{m}}(\hat{\sigma}) / \partial \hat{\sigma}
\end{array}\right.
$$

with their increments

$$
\left\{\begin{aligned}
\mathrm{d} \boldsymbol{\varepsilon}^{\mathrm{M}}= & {\left[\partial^{2} \phi_{\mathrm{M}}\left(\hat{\boldsymbol{\sigma}}, H_{\mathrm{M}}\right) / \partial \hat{\boldsymbol{\sigma}} \otimes \partial \hat{\boldsymbol{\sigma}}\right]: \mathrm{d} \hat{\boldsymbol{\sigma}} } \\
& +\boldsymbol{\varepsilon}^{\mathrm{M}}\left(\hat{\boldsymbol{\sigma}}, H_{\mathrm{M}}+\delta H_{\mathrm{M}}\right)-\boldsymbol{\varepsilon}^{\mathrm{M}}\left(\hat{\boldsymbol{\sigma}}, H_{\mathrm{M}}\right), \\
-\mathrm{d} S_{\mathrm{M}}= & {\left[\partial^{2} \phi_{\mathrm{f}}\left(\tilde{s}_{\mathrm{c}}, H_{\mathrm{f}}\right) / \partial \tilde{s}_{\mathrm{c}}^{2}\right] \mathrm{d} \tilde{s}_{\mathrm{c}} } \\
& -S_{\mathrm{M}}\left(\tilde{s}_{\mathrm{c}}, H_{\mathrm{f}}+\delta H_{\mathrm{f}}\right)+S_{\mathrm{M}}\left(\tilde{s}_{\mathrm{c}}, H_{\mathrm{f}}\right), \\
\mathrm{d} \varepsilon^{\mathrm{m}}= & {\left[\partial^{2} \phi_{\mathrm{m}}(\hat{\sigma}) / \partial \hat{\sigma}^{2}\right] \mathrm{d} \hat{\sigma} . }
\end{aligned}\right.
$$

With eqs. (29) and (30), the plastic increments can be further written as

$$
\left\{\begin{aligned}
\delta \boldsymbol{\varepsilon}^{\mathrm{Mp}} & =\boldsymbol{\varepsilon}^{\mathrm{M}}\left(\hat{\boldsymbol{\sigma}}, H_{\mathrm{M}}+\delta H_{\mathrm{M}}\right)-\boldsymbol{\varepsilon}^{\mathrm{M}}\left(\hat{\boldsymbol{\sigma}}, H_{\mathrm{M}}\right) \\
& =\partial \phi_{\mathrm{M}}\left(\hat{\boldsymbol{\sigma}}, H_{\mathrm{M}}+\delta H_{\mathrm{M}}\right) / \partial \hat{\boldsymbol{\sigma}}-\partial \phi_{\mathrm{M}}\left(\hat{\boldsymbol{\sigma}}, H_{\mathrm{M}}\right) / \partial \hat{\boldsymbol{\sigma}} \\
& =\partial\left(\zeta_{\mathrm{i}}^{\mathrm{M}} \delta \xi_{\mathrm{i}}^{\mathrm{M}}\right) / \partial \hat{\boldsymbol{\sigma}}, \\
-\delta S_{\mathrm{M}}^{\mathrm{p}} & =-S_{\mathrm{M}}\left(\tilde{s}_{\mathrm{c}}, H_{\mathrm{f}}+\delta H_{\mathrm{f}}\right)+S_{\mathrm{M}}\left(\tilde{s}_{\mathrm{c}}, H_{\mathrm{f}}\right) \\
& =\partial \phi_{\mathrm{f}}\left(\tilde{s}_{\mathrm{c}}, H_{\mathrm{f}}+\delta H_{\mathrm{f}}\right) / \partial \tilde{s}_{\mathrm{c}}-\partial \phi_{\mathrm{f}}\left(\tilde{s}_{\mathrm{c}}, H_{\mathrm{f}}\right) / \partial \tilde{s}_{\mathrm{c}} \\
& =\partial\left(\zeta_{\mathrm{i}}^{\mathrm{f}} \delta \xi_{\mathrm{i}}^{\mathrm{f}}\right) / \partial \tilde{s}_{\mathrm{c}} .
\end{aligned}\right.
$$

The elastic increments are then defined by

$$
\left\{\begin{aligned}
\delta \boldsymbol{\varepsilon}^{\mathrm{Me}} & =\delta \boldsymbol{\varepsilon}^{\mathrm{M}}-\delta \boldsymbol{\varepsilon}^{\mathrm{Mp}}=\left[\partial^{2} \phi_{\mathrm{M}}\left(\hat{\boldsymbol{\sigma}}, H_{s}\right) / \partial \hat{\boldsymbol{\sigma}} \otimes \partial \hat{\boldsymbol{\sigma}}\right]: \mathrm{d} \hat{\boldsymbol{\sigma}} \\
& =\boldsymbol{M}_{\mathrm{M}}: \mathrm{d} \hat{\boldsymbol{\sigma}} \\
-\delta S_{\mathrm{M}}^{\mathrm{e}} & =-\mathrm{d} S_{\mathrm{M}}+\delta S_{\mathrm{M}}^{\mathrm{p}}=\left[\partial^{2} \phi_{\mathrm{f}}\left(\tilde{s}_{\mathrm{c}}, H_{\mathrm{f}}\right) / \partial \tilde{s}_{\mathrm{c}}^{2}\right] \mathrm{d} \tilde{s}_{\mathrm{c}} \\
& =M_{\mathrm{f}} \mathrm{d} \tilde{s}_{\mathrm{c}}, \\
\mathrm{d} \varepsilon^{\mathrm{m}}= & {\left[\partial^{2} \phi_{\mathrm{m}}(\hat{\sigma}) / \partial \hat{\sigma}^{2}\right] \mathrm{d} \hat{\sigma} } \\
= & M_{\mathrm{m}} \mathrm{d} \hat{\sigma} .
\end{aligned}\right.
$$

In fact, the plastic work can generally be divided into two portions: one is dissipated and the other is stored as a locked energy [14]. Thus substituting eq. (32) into the increments of the Helmholtz functions eq. (26) yields

$$
\left\{\begin{array}{l}
\mathrm{d} \psi_{\mathrm{M}}=\hat{\boldsymbol{\sigma}}: \delta \varepsilon^{\mathrm{Me}}+\boldsymbol{\alpha}: \delta \varepsilon^{\mathrm{Mp}}+\widehat{\boldsymbol{\sigma}}_{\mathrm{D}}: \delta \varepsilon^{\mathrm{Mp}}-\zeta_{\mathrm{i}}^{\mathrm{M}} \delta \xi_{\mathrm{i}}^{\mathrm{M}}, \\
\mathrm{d} \psi_{\mathrm{f}}=-\tilde{s}_{\mathrm{c}} \delta S_{\mathrm{M}}^{\mathrm{e}}-\beta \delta S_{\mathrm{M}}^{\mathrm{p}}-\tilde{s}_{\mathrm{cD}} \delta S_{\mathrm{M}}^{\mathrm{p}}-\zeta_{\mathrm{i}}^{\mathrm{f}} \delta \xi_{\mathrm{i}}^{\mathrm{f}}, \\
\mathrm{d} \psi_{\mathrm{m}}=\hat{\sigma} \mathrm{d} \varepsilon^{\mathrm{m}},
\end{array}\right.
$$

where $\alpha$ and $\beta$ are termed the back stresses; $\overline{\boldsymbol{\sigma}}_{\mathrm{D}}$ and $\tilde{\boldsymbol{s}}_{\mathrm{cD}}$ are termed the dissipative stresses; and the additive decompositions $\hat{\sigma}=\hat{\sigma}_{\mathrm{D}}+\alpha$ and $\tilde{s}_{\mathrm{c}}=\tilde{s}_{\mathrm{cD}}+\beta$ have been invoked.

Eqs. (24) and (31) demonstrate that the changes of the internal variables and the plastic strain increments are related to the internal structural rearrangements, which can be further written, by incremental linearity, as

$$
\left\{\begin{array}{l}
\delta \xi_{\mathrm{i}}^{\mathrm{M}}=r_{\mathrm{i}}^{\mathrm{M}}\left\langle L_{\mathrm{M}}\right\rangle \\
\delta \xi_{\mathrm{i}}^{\mathrm{f}}=r_{\mathrm{i}}^{\mathrm{f}}\left\langle L_{\mathrm{f}}\right\rangle
\end{array}, \quad\left\{\begin{array}{l}
\delta \boldsymbol{\varepsilon}^{\mathrm{Mp}}=\boldsymbol{m}\left\langle L_{\mathrm{M}}\right\rangle, \\
-\delta S_{\mathrm{M}}^{\mathrm{p}}=-\operatorname{sgn}\left(\delta S_{\mathrm{M}}^{\mathrm{p}}\right)\left\langle L_{\mathrm{f}}\right\rangle,
\end{array}\right.\right.
$$

where $\boldsymbol{m}$ is a second-order tensor that carries the information on the direction of the macrostructural plastic strain increment; $\operatorname{sgn}(\cdot)$ is a step function, and $\operatorname{sgn}\left(\delta S_{\mathrm{M}}^{\mathrm{p}}\right)=1,0$ or -1 for a positive, null or negative $\delta S_{\mathrm{M}}^{\mathrm{p}}$ respectively; $r_{\mathrm{i}}^{\alpha}$ as a scaled version of $\delta \xi_{i}^{\alpha}$. Substituting eq. (34) into eq. (33) yields the yield functions in the dissipative stress space

$$
\left\{\begin{array}{l}
f_{\mathrm{M}}\left(\hat{\boldsymbol{\sigma}}_{\mathrm{D}}, \hat{\boldsymbol{\sigma}}, H_{\mathrm{M}}\right)=\boldsymbol{m}: \hat{\boldsymbol{\sigma}}_{\mathrm{D}}-r_{\mathrm{i}}^{\mathrm{M}} \zeta_{\mathrm{i}}^{\mathrm{M}}=0, \\
f_{\mathrm{f}}\left(\tilde{s}_{\mathrm{cD}}, \tilde{s}, H_{\mathrm{f}}\right)=-\tilde{s}_{\mathrm{cD}} \operatorname{sgn}\left(\delta S_{\mathrm{M}}^{\mathrm{p}}\right)-r_{\mathrm{i}}^{\mathrm{f}} \zeta_{\mathrm{i}}^{\mathrm{f}}=0 .
\end{array}\right.
$$

Based on the relation between the true stresses and the dissipative stresses, the yield functions in the true stress space are given by

$$
\left\{\begin{array}{l}
f_{\mathrm{M}}\left(\hat{\boldsymbol{\sigma}}, H_{\mathrm{M}}\right)=f_{\mathrm{M}}\left[\hat{\boldsymbol{\sigma}}-\boldsymbol{\alpha}\left(\hat{\boldsymbol{\sigma}}, H_{\mathrm{M}}\right), \hat{\boldsymbol{\sigma}}, H_{\mathrm{M}}\right]=0, \\
f_{\mathrm{f}}\left(\tilde{s}_{\mathrm{c}}, H_{\mathrm{f}}\right)=f_{\mathrm{f}}\left[\tilde{s}_{\mathrm{c}}-\beta\left(\tilde{s}_{\mathrm{c}}, H_{\mathrm{f}}\right), \tilde{s}_{\mathrm{c}}, H_{\mathrm{f}}\right]=0 .
\end{array}\right.
$$

The conditions for consistency of the yield functions are given by

$$
\begin{aligned}
& \int \mathrm{d} f_{\mathrm{M}}=\frac{\partial f_{\mathrm{M}}}{\partial \hat{\boldsymbol{\sigma}}}: \mathrm{d} \hat{\boldsymbol{\sigma}}+f_{\mathrm{M}}\left(\hat{\boldsymbol{\sigma}}, H_{\mathrm{M}}+\delta H_{\mathrm{M}}\right)-f_{\mathrm{M}}\left(\hat{\boldsymbol{\sigma}}, H_{\mathrm{M}}\right) \\
& =\mathrm{d} f_{\mathrm{M}}=\frac{\partial f_{\mathrm{M}}}{\partial(\hat{\boldsymbol{\sigma}}-\boldsymbol{\alpha})}: \mathrm{d} \hat{\boldsymbol{\sigma}}-\frac{\partial f_{\mathrm{M}}}{\partial(\hat{\boldsymbol{\sigma}}-\boldsymbol{\alpha})}: \frac{\partial \boldsymbol{\alpha}}{\partial \hat{\boldsymbol{\sigma}}}: \mathrm{d} \hat{\boldsymbol{\sigma}} \\
& +\frac{\partial f_{\mathrm{M}}}{\partial \hat{\boldsymbol{\sigma}}}: \mathrm{d} \hat{\boldsymbol{\sigma}}-\frac{\partial f_{\mathrm{M}}}{\partial(\hat{\boldsymbol{\sigma}}-\boldsymbol{\alpha})}: \delta \boldsymbol{\alpha}^{\mathrm{p}}+\delta f_{\mathrm{M}}^{\mathrm{p}} \\
& =\left[\boldsymbol{m}-\boldsymbol{m}:(\partial \boldsymbol{\alpha} / \partial \hat{\boldsymbol{\sigma}})+\partial f_{\mathrm{M}} / \partial \hat{\boldsymbol{\sigma}}\right]: \mathrm{d} \hat{\boldsymbol{\sigma}} \\
& +\left(\delta f_{\mathrm{M}}^{\mathrm{p}}-\boldsymbol{m}: \delta \boldsymbol{\alpha}^{\mathrm{p}}\right)=0, \\
& \mathrm{~d} f_{\mathrm{f}}=\frac{\partial f_{\mathrm{f}}}{\partial \tilde{s}_{\mathrm{c}}} \mathrm{d} \tilde{s}_{\mathrm{c}}+f_{\mathrm{f}}\left(\tilde{s}_{\mathrm{c}}, H_{\mathrm{f}}+\delta H_{\mathrm{f}}\right)-f_{\mathrm{f}}\left(\tilde{s}_{\mathrm{c}}, H_{\mathrm{f}}\right) \\
& =\mathrm{d} f_{\mathrm{f}}=\frac{\partial f_{\mathrm{f}}}{\partial\left(\tilde{s}_{\mathrm{c}}-\beta\right)} \mathrm{d} \tilde{s}_{\mathrm{c}}-\frac{\partial f_{\mathrm{f}}}{\partial\left(\tilde{s}_{\mathrm{c}}-\beta\right)} \frac{\partial \beta}{\partial \tilde{s}_{\mathrm{c}}} \mathrm{d} \tilde{s}_{\mathrm{c}} \\
& +\frac{\partial f_{\mathrm{f}}}{\partial \tilde{s}_{\mathrm{c}}} \mathrm{d} \tilde{s}_{\mathrm{c}}-\frac{\partial f_{\mathrm{f}}}{\partial\left(\tilde{s}_{\mathrm{c}}-\beta\right)} \delta \beta^{\mathrm{p}}+\delta f_{\mathrm{f}}^{\mathrm{p}} \\
& =\left[-\operatorname{sgn}\left(\delta S_{\mathrm{M}}^{\mathrm{p}}\right)+\operatorname{sgn}\left(\delta S_{\mathrm{M}}^{\mathrm{p}}\right)\left(\partial \beta / \partial \tilde{s}_{\mathrm{c}}\right)+\partial f_{\mathrm{f}} / \partial \tilde{s}_{\mathrm{c}}\right] \mathrm{d} \tilde{s}_{\mathrm{c}} \\
& +\left[\delta f_{\mathrm{f}}^{\mathrm{p}}+\operatorname{sgn}\left(\delta S_{\mathrm{M}}^{\mathrm{p}}\right) \delta \beta^{\mathrm{p}}\right]=0 .
\end{aligned}
$$


in which

$$
\begin{aligned}
& \left\{\begin{array}{l}
\delta \boldsymbol{\alpha}^{\mathrm{p}}=\boldsymbol{\alpha}\left(\hat{\boldsymbol{\sigma}}, H_{\mathrm{M}}+\delta H_{\mathrm{M}}\right)-\boldsymbol{\alpha}\left(\hat{\boldsymbol{\sigma}}, H_{\mathrm{M}}\right), \\
\delta \beta^{\mathrm{p}}=\beta\left(\tilde{s}_{\mathrm{c}}, H_{\mathrm{f}}+\delta H_{\mathrm{f}}\right)-\beta\left(\tilde{s}_{\mathrm{c}}, H_{\mathrm{f}}\right),
\end{array}\right. \\
& \left\{\begin{array}{l}
\delta f_{\mathrm{M}}^{\mathrm{p}}=f_{\mathrm{M}}^{\mathrm{p}}\left(\boldsymbol{\sigma}_{\mathrm{D}}, \hat{\boldsymbol{\sigma}}, H_{\mathrm{M}}+\delta H_{\mathrm{M}}\right)-f_{\mathrm{M}}^{\mathrm{p}}\left(\boldsymbol{\sigma}_{\mathrm{D}}, \hat{\boldsymbol{\sigma}}, H_{\mathrm{M}}\right), \\
\delta f_{\mathrm{f}}^{\mathrm{p}}=f_{\mathrm{f}}^{\mathrm{p}}\left(\tilde{s}_{\mathrm{cD}}, \tilde{s}_{\mathrm{c}}, H_{\mathrm{f}}+\delta H_{\mathrm{f}}\right)-f_{\mathrm{f}}^{\mathrm{p}}\left(\tilde{s}_{\mathrm{cD}}, \tilde{s}_{\mathrm{c}}, H_{\mathrm{f}}\right) .
\end{array}\right.
\end{aligned}
$$

Substituting eqs. (22) and (34) into eq. (38), the evolutions of $\alpha$ and $f_{\mathrm{M}}$ due to $\delta H_{\mathrm{M}}$, as well as $\beta$ and $f_{\mathrm{f}}$ due to $\delta H_{\mathrm{f}}$ can now be written as

$$
\begin{aligned}
& \left\{\begin{array}{l}
\delta \boldsymbol{\alpha}^{\mathrm{p}}=\boldsymbol{n}_{\mathrm{M}}\left\langle L_{\mathrm{M}}\right\rangle+\boldsymbol{n}_{\mathrm{f}} \delta S_{\mathrm{M}}^{\mathrm{p}}+\boldsymbol{n}_{\mathrm{m}} \mathrm{d} \varepsilon^{\mathrm{m}}, \\
\delta \beta^{\mathrm{p}}=b_{\mathrm{f}}\left\langle L_{\mathrm{f}}\right\rangle+\boldsymbol{b}_{\mathrm{M}}: \delta \varepsilon^{\mathrm{Mp}},
\end{array}\right. \\
& \left\{\begin{array}{l}
\delta f_{\mathrm{M}}^{\mathrm{p}}=z_{\mathrm{M}}\left\langle L_{\mathrm{M}}\right\rangle+z_{\mathrm{f}} \delta S_{\mathrm{M}}^{\mathrm{p}}+z_{\mathrm{m}} \mathrm{d} \varepsilon^{\mathrm{m}}, \\
\delta f_{\mathrm{f}}^{\mathrm{p}}=g_{\mathrm{f}}\left\langle L_{\mathrm{f}}\right\rangle+\boldsymbol{g}_{\mathrm{M}}: \delta \varepsilon^{\mathrm{Mp}} .
\end{array}\right.
\end{aligned}
$$

Substituting eq. (39) into eq. (37) yields the following form of the consistency conditions in the true stress space:

$$
\left\{\begin{array}{l}
\mathrm{d} f_{\mathrm{sM}}=\boldsymbol{\mu}: \mathrm{d} \hat{\boldsymbol{\sigma}}-K_{\mathrm{p}}^{\prime}\left\langle L_{\mathrm{M}}\right\rangle-K_{\mathrm{p}}^{\prime \prime} \delta S_{\mathrm{M}}^{\mathrm{p}}-K_{\mathrm{p}}^{\prime \prime \prime} \mathrm{d} \varepsilon^{\mathrm{m}}=0, \\
\mathrm{~d} f_{\mathrm{f}}=\mathrm{d} \tilde{s}_{\mathrm{c}}-H_{\mathrm{p}}^{\prime}\left\langle L_{\mathrm{f}}\right\rangle-H_{\mathrm{p}}^{\prime \prime}: \delta \varepsilon^{\mathrm{Mp}}=0 .
\end{array}\right.
$$

where

$$
\begin{aligned}
& \left\{\begin{array}{l}
\boldsymbol{\mu}=\partial f_{\mathrm{M}} / \partial \hat{\boldsymbol{\sigma}}, \\
K_{\mathrm{p}}^{\prime}=\boldsymbol{m}: \boldsymbol{n}_{\mathrm{M}}-z_{\mathrm{M}}, \\
K_{\mathrm{p}}^{\prime \prime}=\boldsymbol{m}: \boldsymbol{n}_{\mathrm{f}}-z_{\mathrm{f}}, \\
K_{\mathrm{p}}^{\prime \prime \prime}=\boldsymbol{m}: \boldsymbol{n}_{\mathrm{m}}-z_{\mathrm{m}},
\end{array}\right. \\
& \left\{\begin{array}{l}
H_{\mathrm{p}}^{\prime}=-\left[\operatorname{sgn}\left(\delta S_{\mathrm{M}}^{\mathrm{p}}\right) b_{\mathrm{f}}+g_{\mathrm{f}}\right] /\left(\frac{\partial f_{\mathrm{f}}}{\partial \tilde{s}_{\mathrm{c}}}\right), \\
\boldsymbol{H}_{\mathrm{p}}^{\prime \prime}=-\left[\operatorname{sgn}\left(\delta S_{\mathrm{M}}^{\mathrm{p}}\right) \boldsymbol{b}_{\mathrm{M}}+\boldsymbol{g}_{\mathrm{M}}\right] /\left(\frac{\partial f_{\mathrm{f}}}{\partial \tilde{s}_{\mathrm{c}}}\right) .
\end{array}\right.
\end{aligned}
$$

From the consistency conditions eq. (40), the loading indexes are given by

$$
\left\{\begin{array}{l}
L_{\mathrm{M}}=\left(\boldsymbol{\mu} / K_{\mathrm{p}}^{\prime}\right): \mathrm{d} \hat{\boldsymbol{\sigma}}-\left(K_{p}^{\prime \prime} / K_{p}^{\prime}\right) \delta S_{\mathrm{M}}^{\mathrm{p}}-\left(K_{p}^{\prime \prime \prime} / K_{p}^{\prime}\right) \mathrm{d} \varepsilon^{m}, \\
L_{\mathrm{f}}=\left(1 / H_{\mathrm{p}}^{\prime}\right) \mathrm{d} \tilde{s}_{c}-\left(H_{\mathrm{p}}^{\prime \prime} / H_{\mathrm{p}}^{\prime}\right): \delta \boldsymbol{\varepsilon}^{\mathrm{Mp}} .
\end{array}\right.
$$

Then the plastic strain increments are given by

$$
\left\{\begin{aligned}
\delta \boldsymbol{\varepsilon}^{\mathrm{Mp}}= & h\left(L_{\mathrm{M}}\right)\left\{\left[(\boldsymbol{m} \otimes \boldsymbol{\mu}) / K_{\mathrm{p}}^{\prime}\right]: \mathrm{d} \hat{\boldsymbol{\sigma}}-\boldsymbol{m}\left(K_{\mathrm{p}}^{\prime \prime} / K_{\mathrm{p}}^{\prime}\right) \delta S_{\mathrm{M}}^{\mathrm{p}}\right. \\
& \left.-\boldsymbol{m}\left(K_{\mathrm{p}}^{\prime \prime \prime} / K_{\mathrm{p}}^{\prime}\right) \mathrm{d} \varepsilon^{\mathrm{m}}\right\}, \\
-\delta S_{\mathrm{M}}^{\mathrm{p}}= & -h\left(L_{\mathrm{f}}\right) \operatorname{sgn}\left(\delta S_{\mathrm{M}}^{\mathrm{p}}\right) \\
& \times\left[\left(1 / H_{\mathrm{p}}^{\prime}\right) \mathrm{d} \tilde{s}_{\mathrm{c}}-\left(\boldsymbol{H}_{\mathrm{p}}^{\prime \prime} / H_{\mathrm{p}}^{\prime}\right): \delta \boldsymbol{\varepsilon}^{\mathrm{Mp}}\right],
\end{aligned}\right.
$$

where $h(\cdot)$ denotes the Heaviside step function, if $\cdot>0$, $h(\cdot)=1$, else $h(\cdot)=0$. Combining eqs. (32) and (43) yields

$$
\left\{\begin{aligned}
\mathrm{d} \boldsymbol{\varepsilon}^{\mathrm{M}}= & {\left[\boldsymbol{M}_{\mathrm{M}}+h\left(L_{\mathrm{M}}\right)\left(\boldsymbol{m} \otimes \boldsymbol{\mu} / K_{\mathrm{p}}^{\prime}\right)\right]: \mathrm{d} \hat{\boldsymbol{\sigma}}-h\left(L_{\mathrm{M}}\right) } \\
& \times \boldsymbol{m}\left(K_{\mathrm{p}}^{\prime \prime} / K_{\mathrm{p}}^{\prime}\right) \delta S_{\mathrm{M}}^{\mathrm{p}}-h\left(L_{\mathrm{M}}\right) \boldsymbol{m}\left(K_{\mathrm{p}}^{\prime \prime \prime} / K_{\mathrm{p}}^{\prime}\right) \mathrm{d} \varepsilon^{\mathrm{m}}, \\
-\mathrm{d} S_{\mathrm{M}}= & {\left[M_{\mathrm{f}}-h\left(L_{\mathrm{f}}\right) \operatorname{sgn}\left(\delta S_{\mathrm{M}}^{\mathrm{p}}\right)\left(1 / H_{\mathrm{p}}^{\prime}\right)\right] \mathrm{d} \tilde{s}_{\mathrm{c}} } \\
& +h\left(L_{\mathrm{f}}\right) \operatorname{sgn}\left(\delta S_{\mathrm{M}}^{\mathrm{p}}\right)\left(H_{\mathrm{p}}^{\prime \prime} / H_{\mathrm{p}}^{\prime}\right): \delta \boldsymbol{\varepsilon}^{\mathrm{Mp}}, \\
\mathrm{d} \varepsilon^{\mathrm{m}}= & M_{\mathrm{m}} \mathrm{d} \hat{\sigma} .
\end{aligned}\right.
$$

The set of incremental stress-strain equations is expressed by eq. (44). It shows that the each phase of the unsaturated expansive soils with double porosity is subjected to mutual influence. Based on the thermodynamics-based modelling framework, we have further proposed a constitutive model which is able to quantitatively predict the capillary hysteresis and mechanical behaviour in unsaturated expansive soils [33].

\section{Discussion}

Unsaturated expansive soils, such as natural and compacted expansive soils or bentonite pellet mixtures, possess two significant characteristics: (1) a pore size distribution with at least two dominant values of porosity; (2) a more obvious interaction of pore water and soil particle surfaces. In this paper, based on the porous media theory, the specific expressions of the total stress and the input work are proposed. The energy-conjugate variables are further derived, which are $\left\langle\hat{\sigma}, \dot{\boldsymbol{\varepsilon}}^{\mathrm{M}}\right\rangle,\left\langle\tilde{s}_{\mathrm{c}}, \dot{S}_{\mathrm{M}}\right\rangle$ and $\left\langle\hat{\sigma}, \dot{\varepsilon}^{\mathrm{m}}\right\rangle$. We make distinctions between the effects of the capillary water and the adsorbed water on the soil behaviour, as well as between the deformations of micro- and macrostructure.

According to the derived work equation and the workenergy-dissipation relations for an open multiphase thermodynamic system, we establish the thermodynamics-based modelling framework for unsaturated expansive soils with double porosity. Within the proposed framework, we have taken into consideration the effect of the microstructural volume change on the deformation of macrostructure and the coupling of water retention behaviour and stress-strain behaviour.

This work was supported by the National Natural Science Foundation of China (51278047), the National Basic Research Program of China (2010CB$732100)$ and Beijing Municipal Natural Science Foundation (8112024).

1 Barenblatt G I, Zheltov I P, Kochina I N. Basic concepts in the theory of seepage of homogeneous liquids in fissured rocks. J Appl Math Mech, 1960, 24: 1286-1303

2 Delage P, Tessier D, Marcel-Audiguier M. Use of the cryoscan apparatus for observation of freeze-fractured planes of a sensitive Quebec clay in scanning electron microscopy. Can Geotech J, 1982, 19: 111114

3 Sridharan A, Altschaeffl A G, Diamond S. Pore size distribution studies. J Soil Mech Found Div, 1971, 97: 771-787 
4 Hoffmann C. Hydromechanics characterization of bentonite pellets mixtures. The study of experimental and constitutive (in Spanish). Dissertation for Doctoral Degree. Barcelona: Polytechnic University of Catalonia, 2005

5 Delage P, Lefebvre G. Study of the structure of a sensitive Champlain clay and of its evolution during consolidation. Can Geotech J, 1984, 21: 21-35

6 Juang C H, Holtz R D. Fabric, pore size distribution, and permeability of sandy soils. J Geotech Engrg, 1986, 112: 855-868

7 Romero E, Della Vecchia G, Jommi C. An insight into the water retention properties of compacted clayey soils. Géotechnique, 2011, 61: 313-328

8 Gens A, Alonso E E. A framework for the behaviour of unsaturated expansive clays. Can Geotech J, 1992, 29: 1013-1032

9 Alonso E E, Vaunat J, Gens A. Modelling the mechanical behaviour of expansive clays. Engng Geol, 1999, 54: 173-183

10 Lu Z H, Wang Q M, Chen Z H. Research on the constitutive model of unsaturated expansive soil (in Chinese). Undergr Sp, 2001, 21: 379-385

11 Cao X S. Elastoplastic constitutive model of unsaturated expansive soils (in Chinese). Chin J Geotech Eng, 2005, 27: 832-836

12 Sánchez M, Gens A, Guimarães L Do N, et al. A double structure generalized plasticity model for expansive materials. Int J Numer Anal Meth Geomech, 2005, 29: 751-787

13 Ziegler $\mathrm{H}$, Wehrli C. The derivation of constitutive relations from the free energy and the dissipation function. Adv Appl Mech, 1987, 25: $183-238$

14 Collins I F, Houlsby G T. Application of thermomechanical principles to the modelling of geotechnical materials. Proc R Soc Lond A, 1997, 453: 1975-2001

15 Puzrin A M, Houlsby G T. A thermomechanical framework for rate-independent dissipative materials with internal functions. Int $\mathrm{J}$ Plasticity, 2001, 17: 1147-1165

16 Li X S. Thermodynamics-based constitutive framework for unsaturated soils. 1: Theory. Géotechnique, 2007, 57: 411-422

17 Li X S. Thermodynamics-based constitutive framework for unsaturated soils. 2: A basic triaxial model. Géotechnique, 2007, 57: 423435

18 Zhao C G, Liu Y. Continuum porous medium soil mechanics and its application in constitutive relationship of unsaturated soils (in Chinese). Chin J Geotech Engng, 2009, 31: 1324-1335

19 Liu Y, Zhao C G, Cai G Q, et al. Constitutive modelling for unsaturated soils considering gas hardening effect. Chin Sci Bull, 2011, 56, 1739-1745

20 Derjaguin B V, Churaev N V, Muller V M. Surface Forces. New York: Plenum Press, 1987

21 Tuller M, Or D, Dudley L M. Adsorption and capillary condensation in porous media: Liquid retention and interfacial configurations in angular pores. Water Resour Res, 1999, 35: 1949-1964

22 Gens A. Soil-environment interactions in geotechnical engineering. Géotechnique, 2010, 60: 3-74

23 Lu N, Likos W J. Unsaturated Soil Mechanics. New York: John Wiley, 2004

24 Lu N, Likos W J. Suction stress characteristic curve for unsaturated soils. J Geotech Geoenviron Eng, 2006, 132: 131-142

25 Borja R I, Koliji A. On the effective stress in unsaturated porous continua with double porosity. J Mech Phys Solids, 2009, 57: 1182-1193

26 Baker R, Frydman S. Unsaturated soil mechanics: Critical review of physical foundations. Engng Geol, 2009, 106: 26-39

27 Houlsby G T. The work input to an unsaturated granular material. Géotechnique, 1997, 47: 193-196

28 Zhao C G, Liu Y, Gao F P. Work and energy equations and the principle of generalized effective stress for unsaturated soils. Int J Numer Anal Meth Geomech, 2010, 34: 920-936

29 Cai G Q, Zhao C G, Liu Y, et al. A nonlinear multi-field coupled model for soils. Sci China E: Tech Sci, 2011, 54: 1300-1314

30 Mitchell J K, Soga K. Fundamentals of Soil Behavior. 3rd ed. New York: John Wiley, 2005

31 Wheeler S J, Sharma R S, Buisson M S R. Coupling of hydraulic hysteresis and stress-strain behaviour in unsaturated soils. Géotechnique, 2003, 53: 41-54

32 Rice J R. Inelastic constitutive relations for solids: An internal-variable theory and its application to metal plasticity. J Mech Phys Solids, 1971, 19: 433-455

33 Li J, Zhao C G, Huang Q D. The constitutive modelling with double scale pore structure for coupling of capillary hysteresis and stressstrain behaviour in unsaturated expansive soil (in Chinese). Chin J Geotech Eng, 2012, 34: 2127-2133

Open Access This article is distributed under the terms of the Creative Commons Attribution License which permits any use, distribution, and reproduction in any medium, provided the original author(s) and source are credited. 